\title{
Targeting bladder tumor cells in voided urine of Chinese patients with FITC-CSNRDARRC peptide ligand
}

\section{Xing-You Jia' \\ Qi Yu² \\ Zhe-Hui Zhang ${ }^{3}$ \\ Xiao-Feng Yang'}

'School of the First Clinical Hospital, Shanxi Medical University, Taiyuan, Shanxi, China; ${ }^{2}$ Department of Information Management, Shanxi Medical University, Taiyuan, Shanxi, China; ${ }^{3}$ Research Center for Philosophy of Science and Technology, Shanxi University, Taiyuan, Shanxi, China
Correspondence: Xiao-Feng Yang Department of Urology, First Affiliated Hospital of Shanxi Medical University, 85 Jiefang South Road, Taiyuan, China $\mathrm{Tel}+86035$ I 4639826

Fax +86 035 I 228 I I0।

Email yxfylq@।63.com
This article was published in the following Dove Press journal:

OncoTargets and Therapy

5 May 2012

Number of times this article has been viewed

Objective: To study the practicality of the FITC-CSNRDARRC peptide ligand (containing the Cys-Ser-Asn-Arg-Asp-Ala-Arg-Arg-Cys nonapeptide) in diagnosing and monitoring bladder tumors.

Materials and methods: Between March 2011 and September 2011, 80 consecutive patients with radiographic abnormalities, localizing hematuria, other symptoms, or signs were studied using the FITC-CSNRDARRC ligand, urinary cytology (UC), and fluorescence in situ hybridization (FISH). The sensitivity and specificity of these three technologies were determined and compared. Cystoscopy and tissue biopsy were taken as the "gold standards" for bladder tumor diagnosis in this study.

Results: Twenty-nine out of 80 patients were diagnosed with a bladder tumor via histopathological examination. The FITC-CSNRDARRC ligand was positive in 23 out of 29 bladder tumor patients and produced false negatives in six (20.69\%) patients. The UC was positive in six out of 29 bladder tumor patients and produced false negatives in $23(79.31 \%)$ patients. The FISH was positive in 21 out of 29 bladder tumor patients and produced false negatives in eight $(27.59 \%)$ patients. The overall sensitivity as verified by the FITC-CSNRDARRC ligand was much higher than in UC (79.31\% versus $20.69 \%, P<0.001)$ and was slightly higher than in FISH $(79.31 \%$ versus $72.41 \%, P=0.625)$. The sensitivity of FISH was significantly higher than that of UC (72.41\% versus $20.69 \%, P<0.001$ ). Sensitivities of the FITC-CSNRDARRC ligand and UC by grade were $58.33 \%$ versus $8.3 \%$ for low-grade (LG) tumors $(P=0.031)$ and $94.12 \%$ versus $29.41 \%$ for high-grade $(\mathrm{HG})$ tumors $(P=0.003)$, respectively. The advantage was maintained in terms of the detection of invasive tumors between the FITC-CSNRDARRC ligand and UC (90.48\% versus $23.81 \%, P=0.001)$ as well as between FISH and UC ( $85.71 \%$ versus $23.81 \%$, $P=0.003$ ). The specificities for the FITC-CSNRDARRC ligand, UC, and FISH were $100 \%$.

Conclusion: Results show that the FITC-CSNRDARRC ligand is a promising noninvasive tool for diagnosis and surveillance in patients suspected of having a new bladder tumor.

Keywords: bladder tumor, tumor-targeting, FITC-CSNRDARRC ligand, fluorescent probe

Bladder tumors, accounting for 3.3\% of all malignancies, are the ninth most common type of tumor worldwide. ${ }^{1,2}$ Bladder tumor patients are at high risk of recurrence and $7 \%-40 \%$ of patients will develop invasive tumors. ${ }^{3-5}$ The incidence of bladder tumors is also very high in China and has increased year on year. The main concern for doctors is in discerning which techniques should be used to diagnose bladder tumors in their early stages in order to improve the quality of the patients' life. Early diagnosis and the monitoring of disease recurrence play a crucial role in the diagnosis of bladder tumors. 
Optical imaging has been increasingly used to probe protein function and gene expression in live animals. ${ }^{6}$ Peptide targeting of tumors is a new technique used for the diagnosis of bladder tumors. The CSNRDARRC ligand is the most recently developed diagnostic probe for the detection of bladder cancer. $^{7}$

The CSNRDARRC sequence was selected as a synthetic peptide. In the study by Lee et $\mathrm{al}^{7}{ }^{7}$ the fluorescein-conjugated CSNRDARRC peptide selectively bound to human bladder tumor tissue, whereas only negligible binding to normal bladder tissue was observed. In our study, we made a further test of the feasibility of the peptide in detecting tumor cells in urine, with the peptide showing little binding to the urinary cells of patients with inflammation, or to healthy individuals' cells.

Knowledge concerning the genetic changes in bladder tumor cells provides the basis for the development of genetic urine tests. ${ }^{8}$ FISH proved to be an effective method for identifying chromosomal aberrations. ${ }^{9}$ In 2000 , Sokolova et $\mathrm{l}^{10}$ developed what would become the first commercially available FISH probe set for bladder urothelial carcinoma detection in voided urine. The UroVysion FISH detection system (Abbott Molecular, DesPlaines, IL) received Food and Drug Administration (FDA) approval in 2001 for the detection of recurrent tumors in patients with a history of bladder cancer, while the use of the FISH technique is still in the clinical trial stage in China.

UC has a low sensitivity $(<50 \%)$ but a high specificity (>90\%), particularly for LG tumors. ${ }^{11}$ Compared with UC, alternative methods, such as the bladder tumor antigen (BTA) test, the urinary nuclear matrix protein 22 (NMP22), surviving (SV), and telomerase tests have become available, but none of them has yet attained specificity equal to, or better than cytology. ${ }^{11,12}$ The sensitivity and specificity of the most used urinary tumor markers in detecting primary bladder cancer (ie, the tumor markers BTA, NMP22, SV, and telomerase) are far from satisfactory. ${ }^{13-16}$

Cystoscopy, tissue biopsy, and UC are three of the main technologies that are used for bladder tumor diagnosis, but all of them have issues. ${ }^{17,18}$ Cystoscopy and tissue biopsy are invasive techniques, and UC has a low sensitivity, so a simpler and higher sensitivity tumor diagnosis technique is needed. This study examines the FITC-CSNRDARRC ligand, which takes CSNRDARRC as the tumor marker and which has a high specificity for bladder tumor diagnosis. The aim of this study is to compare the specificity and sensitivity of the FITC-CSNRDARRC ligand, UC, and FISH, and to explore the clinical value of the FITC-CSNRDARRC ligand.
Cystoscopy and tissue biopsy were taken as the "gold standards" for bladder tumor diagnosis in this study.

\section{Materials and methods}

Voided urine specimens from 80 consecutive patients (mean age, 67.27 years; standard deviation [SD], 3.25 years; range, 41-88 years; males, 58 cases; females, 22 cases) with radiographic abnormalities, localizing hematuria, other symptoms, or signs were obtained between March 2011 and September 2011.

\section{Urinary cytology}

For each sample, $50 \mathrm{~mL}$ of voided urine was centrifuged at $1300 \mathrm{rpm}$ for 10 minutes at room temperature and then the supernatant was discarded. After pretreatment with $10 \%$ formaldehyde fixative, approximately $30 \mathrm{uL}$ of the cell pellet was dropped onto a standard glass slide. The glass slide was heated for 30 minutes at $56^{\circ} \mathrm{C}$. After Papanicolaou or hematoxylin staining, the cell pellet was examined by microscope.

\section{FISH analysis}

Urine preparation for FISH

A hypotonic solution of potassium chloride $(0.075 \mathrm{M})$ was preheated in a constant temperature bath at $37^{\circ} \mathrm{C}$ for at least 30 minutes. For each sample, $50 \mathrm{~mL}$ of voided urine was centrifuged at $1300 \mathrm{rpm}$ for 10 minutes at room temperature and the supernatant was discarded. This was followed by incubation in a $5 \mathrm{~mL}$ hypotonic solution of potassium chloride for 30 minutes. Fresh Carnoy's fixative $(5 \mathrm{~mL}, 3: 1$ by volume of methanol-glacial acetic acid) was added to the cell pellet, and the pellet was mixed and resuspended. The resulting pellet was centrifuged again and the supernatant was discarded. The process was repeated three times. Approximately $30 \mathrm{uL}$ of cell pellet was dropped onto an APES-coated glass slide (3-aminopropyl-triethoxysilane), which was then air-dried and placed under a microscope in order to label a region with appropriate cellularity. The glass slide was heated for 1 hour at $56^{\circ} \mathrm{C}$.

\section{FISH}

After pretreatment with $2 \times$ sodium saline citrate (SSC) and pepsin at $37^{\circ} \mathrm{C}$ in a water bath with $10 \%$ formaldehyde at room temperature, the slides were dehydrated in $70 \%$, $80 \%$, and $100 \%$ ethanol. Then, $2 \mathrm{uL}$ of probe mixture, which was denatured at $73^{\circ} \mathrm{C}$ for five minutes, was added to each slide and sealed under a small glass coverslip, before overnight hybridization at $42^{\circ} \mathrm{C}$. The procedure was 
followed by washing in $0.4 \times \mathrm{SSC} / 0.3 \%$ Nonidet (NP40) and $2 \times$ SSC. After hybridization, the nuclei counterstaining was achieved with 4',6-diamidino-2-phenylindole (DAPI). Slides were scored for hybridization signals on a cell-bycell basis using a fluorescence microscope.

In every case, the severity of the genetic alteration was defined as the percentage of cells showing cytogenetic abnormalities greater than the cut-off values (ie, the number of cells that showed a gain for at least two of the chromosomes 3, 7, and 17 , or that showed complete deletion of the $p 16(9 p 21)$ locus divided by the total enumerated cells, with the quotient multiplied by 100).

\section{FITC-CSNRDARRC ligand}

The procedure for the urine preparation for the FITC-CSNRDARRC ligand was the same as that of FISH.

The $15 \mathrm{mg} / \mathrm{L}$ solution of FITC-CSNRDARRC ligand in PBS was added to the glass slide and then it was incubated at $4^{\circ} \mathrm{C}$ for 1 hour. After being washed in PBS and air-dried, the nuclei counterstaining was achieved with DAPI. Slides were scored for hybridization signals on a cell-by-cell basis using a fluorescence microscope.

\section{Statistical analysis}

The sensitivities of the FITC-CSNRDARRC ligand, UC, and FISH were determined for patients with biopsy-proven urothelial carcinoma in the bladder, and the specificities were calculated for patients with no histological confirmation of urothelial carcinoma or with negative cystoscopy findings. McNemar's test was used to determine the statistical difference among the use of the three techniques (FITCCSNRDARRC ligand, UC, and FISH). A $P$-value of $<0.05$ was considered to indicate statistical significance. The SPSS 15.0 software package (SPSS, Chicago, IL) was used for statistical analyses.

\section{Results}

A total of 80 voided urine specimens were analyzed in this study: 58 from men and 22 from women. Twenty-nine patients were diagnosed as having a bladder tumor, of which two had pTa-LG disease, three had pTa-HG disease, two had pT1-LG disease, one had pT1-HG disease, three had pT2-LG disease, six had pT2-HG disease, five had pT3-LG disease, four had pT3-HG disease, and three had pT4-HG disease.

\section{FITC-CSNRDARRC ligand}

The FITC-CSNRDARRC ligand was positive in 23 out of 29 bladder tumor patients and produced false negatives in six $(20.69 \%)$ patients. The sensitivities and specificities of the FITC-CSNRDARRC ligand were $79.31 \%$ and $100 \%$, respectively (Table 1 ).

The FITC-CSNRDARRC ligand was produced by TASH Biotechnology Co, Ltd (Shanghai, China). The CSNRDARRC peptide selectively bound to the nuclei of bladder tumor cells. The cells were determined as being positive if there were fluorescent probe signals in the nuclei (Figure 1). ${ }^{19}$

\section{Urinary cytology}

Of the 29 bladder tumor patients, six patients had a positive $\mathrm{UC}$ result and 23 had a false-negative UC result $(79.31 \%)$. The sensitivities and specificities of the UC were $20.69 \%$ and $100 \%$, respectively (Table 1 ).

Results were assessed as positive only in cases where malignancy was detected (Figure 2C). Suspicious and equivocal cases were assessed as negative for the calculations of sensitivity and specificity.

\section{$\mathrm{FISH}$}

The FISH showed positive results in 21 out of 29 bladder tumor patients and produced false negatives in eight $(27.59 \%)$ cases. The sensitivities and specificities of the FISH were $72.41 \%$ and $100 \%$, respectively (Table 1 ).

Table I Detailed comparison of FITC-CSNRDARRC ligand, UC and FISH for the detection of bladder tumor in relation to tumor stages and tumor grades

\begin{tabular}{|c|c|c|c|c|c|c|}
\hline \multirow[t]{2}{*}{ Tumors } & \multicolumn{2}{|l|}{ UC } & \multicolumn{2}{|l|}{ FISH } & \multicolumn{2}{|c|}{ FITC-CSNRDARRC } \\
\hline & $n / N$ & $\%$ & $n / N$ & $\%$ & $\mathrm{n} / \mathrm{N}$ & $\%$ \\
\hline \multicolumn{7}{|l|}{ Grade } \\
\hline Low & $1 / 12$ & 8.3 & $6 / 12$ & 41.67 & $7 / 12$ & 58.33 \\
\hline High & $5 / 17$ & 29.41 & $15 / 17$ & 88.24 & $16 / 17$ & 94.12 \\
\hline \multicolumn{7}{|l|}{ Stage } \\
\hline $\mathrm{pTa}+\mathrm{pTI}$ & $1 / 8$ & 12.5 & $3 / 8$ & 37.5 & $4 / 8$ & 50 \\
\hline $\mathrm{pT} 2-\mathrm{pT} 4$ & $5 / 21$ & 23.81 & $|8 / 2|$ & 85.71 & $19 / 21$ & 90.48 \\
\hline Overall sensitivity & $6 / 29$ & 20.69 & $21 / 29$ & 72.41 & $23 / 29$ & 79.31 \\
\hline Overall specificity & $5|/ 5|$ & 100 & $51 / 51$ & 100 & $5 \mid / 5 I$ & 100 \\
\hline
\end{tabular}

Abbreviations: $\mathrm{FISH}$, fluorescence in situ hybridization; UC, urinary cytology. 


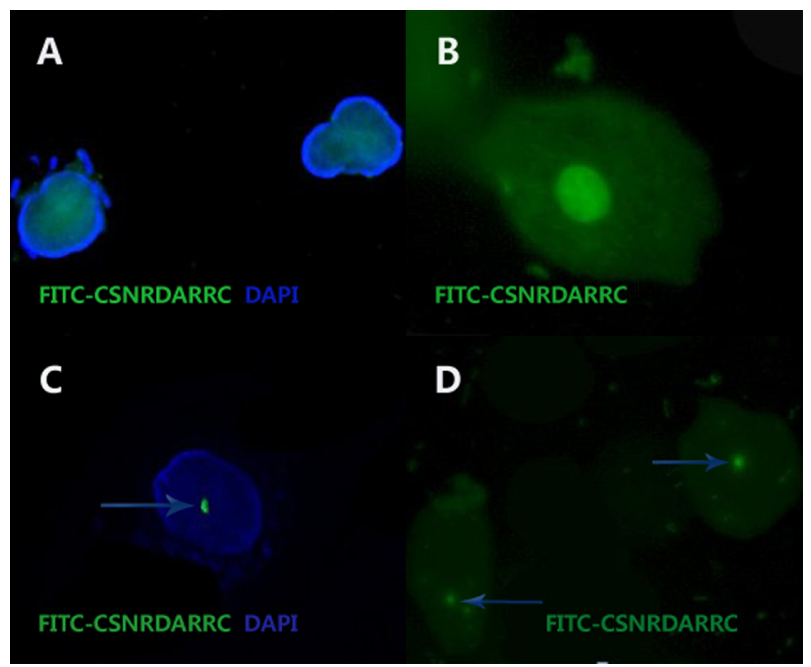

Figure I The FITC-CSNRDARRC ligand. (A and $\mathbf{C}$ ) The nuclei counterstaining was achieved with DAPI. The light colored area in (B) is the nuclei. (D) DAPI was not used in the nuclei.

Notes: The CSNRDARRC peptide is selectively bound to the nuclei of bladder tumor cells. The bright dot pointed at by the arrows indicate where the peptides bind. Magnification $\times 1000$ (A-D).

Abbreviations: DAPI, 4', 6-diamidino-2-phenylindole.

Probes specific for the centromeres of chromosomes 3, 7, 17, and for the $p 16$ locus (GP Medical Technologies, Ltd, Beijing, China) were used in this study (Figure 3). Two DNA probes were mixed together as a set double-target FISH and paired as follows: chromosome 3 and $p 16$ (rhodamine), chromosome 7 and 17 (FITC).

\section{Statistical analysis}

There is a significant difference in bladder tumor diagnosis in terms of the sensitivities of the FITC-CSNRDARRC ligand and UC (79.31\% versus $20.69 \%, P<0.001)$ (Tables 1 and 3 ),

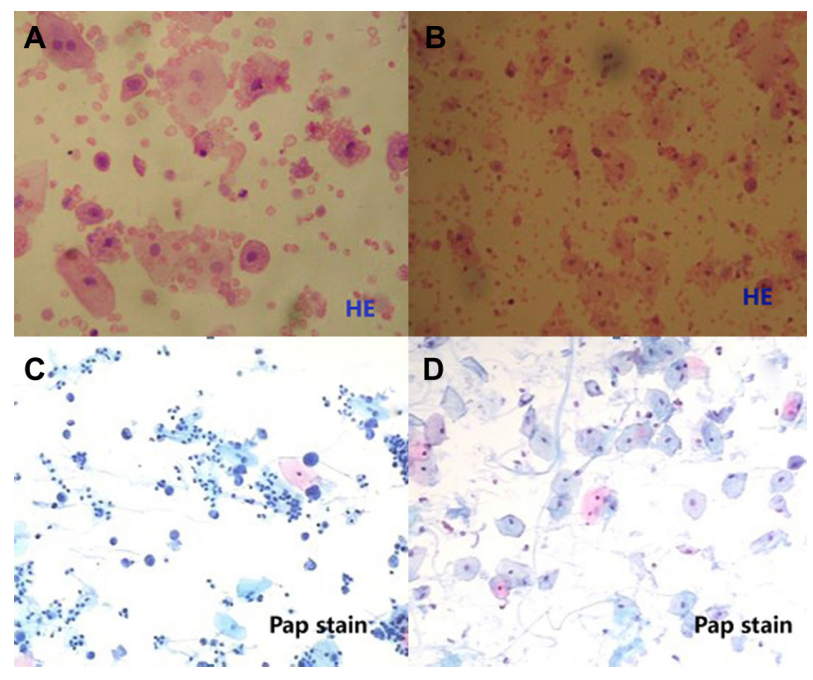

Figure 2 UC. (A and B) Hematoxylin stain, (C and D) Papanicolaou stain. (A, B and D) Three examples of normal cells and an example of tumor cells (C). Note: Magnification $\times 400(\mathbf{A}$ and $\mathbf{D})$ and $\times 200$ (B and C)

Abbreviation: UC, urinary cytology.

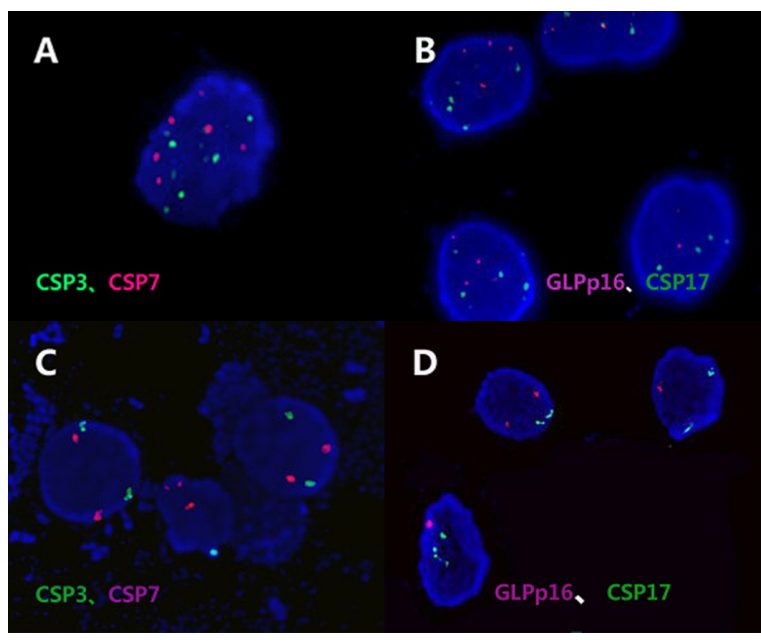

Figure 3 FISH. Chromosome 3 and pl 6 (rhodamine), chromosome 7 and 17 (FITC). (A and B) Example of the same urine specimen cell, in which the percentage of FISH signals are greater than the cut-off value (CSP3, 23\%; CSP7, 21\%; GLPpI6, 7\%; CSPI7,9\%). (C and D) Example of the same urine specimen cell, in which the percentage of FISH signals are lower than the cut-off value (CSP3, 3\%; CSP7, 2\%; GLPpI6, 4\%; CSPI 7, 2\%).

Note: Magnification $\times 1000$ (A-D).

Abbreviation: $\mathrm{FISH}$, fluoresceuce in situ hybridization.

while no significant differences were found between the FITCCSNRDARRC ligand and FISH (79.31\% versus $72.41 \%$, $P=0.625$ ) (Tables 1 and 3). A significant difference was found between the sensitivities of FISH and UC $(72.41 \%$ versus $20.69 \%, P<0.001$ ) (Tables 1 and 3). Meanwhile, the overall specificity for the FITC-CSNRDARRC ligand, UC, and FISH was $100 \%$, which was not statistically significant.

Sensitivities of the FITC-CSNRDARRC ligand and UC by grade were $58.33 \%$ versus $8.3 \%$ for $\mathrm{LG}(P=0.031)$ and $94.12 \%$ versus $29.41 \%$ for $\mathrm{HG}(P=0.003)$ (Tables 1 and 3), respectively, whereas the sensitivities of FISH and UC by grade were $41.67 \%$ versus $8.3 \%$ for LG $(P=0.063)$ (Tables 1 and 3 ) and $88.24 \%$ versus $29.41 \%$ for $\mathrm{HG}$ tumors $(P=0.006)$ (Tables 1 and 3), respectively. Moreover, the advantage is maintained for invasive tumors between the FITC-CSNRDARRC ligand and UC $(90.48 \%$ versus $23.81 \%$, $P=0.001$ ) (Tables 1 and 3 ) as well as between FISH and UC (85.71\% versus $23.81 \%, P=0.003$ ) (Tables 1 and 3 ).

\section{Discussion}

In the present study, we compared the performances of UC, FISH, and the FITC-CSNRDARRC ligand in the detection of bladder tumor cells in voided urine.

The high sensitivity obtained with the use of the FITCCSNRDARRC ligand in the 29 patients with bladder tumors was $79.31 \%$, whereas with FISH it was $72.41 \%$, compared with UC at only a $20.69 \%$ (both $P<0.001$ ) detection rate. No significant difference was found between the FITCCSNRDARRC ligand and FISH $(P=0.625)$. As previously 
Table 2 The predictive values of three methods

\begin{tabular}{llll}
\hline Classification & UC (\%) & FISH (\%) & FITC-CSNRDARRC (\%) \\
\hline PPV & $\mathrm{I}$ & $\mathrm{I}$ & $\mathrm{I}$ \\
NPV & 68.92 & 86.44 & 89.47 \\
\hline
\end{tabular}

Abbreviations: PPV, positive predictive value; NPV, negative predictive value.

described, the sensitivity of FISH was higher for the detection of tumor cells in urine, compared with that of UC. ${ }^{20} \mathrm{In}$ addition, the overall sensitivities of FISH and UC in this study were lower than those reported in several previous studies. Akkad et a $1^{12}$ obtained a sensitivity of $87.5 \%$ for FISH in contrast to the $72.41 \%$ found in our study. A possible explanation for this discrepancy is that there were different distributions of stage and grade in the analyzed samples in each study. The cytology had a sensitivity of $60 \%,{ }^{12}$ while we obtained a low sensitivity of only $20.69 \%$.

Generally, the specificity of FISH is very high when compared to that of other urine tests. ${ }^{21}$ For patients without bladder cancer, we found the same specificity of $100 \%$ for the FITC-CSNRDARRC ligand, FISH, and UC, which was not statistically significant.

In our study, six cases (false-negative rate $=20.69 \%$ ) did not demonstrate the presence of carcinoma with the FITC-CSNRDARRC ligand, eight cases (27.59\%) did not demonstrate this with FISH, and 23 cases (79.31\%) showed no carcinoma presence with UC. The sensitivity can usually be improved by incorporating a washing cytology at different levels of the intubated ureter. However, even this method is not really reliable, with a false-negative rate of up to $39 \% .{ }^{22}$ It has been previously suggested that a falsenegative result represents mostly LG urothelial lesions that do not shed tumor cells into the urine or that do not exhibit the chromosomal alterations that are detected through the assay procedure.

The positive predictive values (PPVs) of the FITC-CSNRDARRC ligand, UC, and FISH in our study were 100\% (Table 2). The PPV that we found for FISH was slightly higher than that in the Marin-Aguileras study ${ }^{23}(100 \%$ versus $95.8 \%$ ). The negative predictive value (NPV) of the
FITC-CSNRDARRC ligand was slightly higher than the FISH $(89.47 \%$ versus $86.44 \%$ ) and was distinctly better than the UC ( $89.47 \%$ versus $68.92 \%$ ) (Table 2$)$, which means that the FITC-CSNRDARRC ligand performed best in terms of excluding bladder tumors. The NPV of FISH was $72 \%$ in the studies mentioned above.

In the present study, two cases had a positive result with the FITC-CSNRDARRC ligand, while all other methods gave negative results. However, we cannot assume that the two cases were real false-positive results. Whether these cases can be considered as positives has yet to be clarified. The actual follow-up was too brief to give a reliable answer. ${ }^{24}$ The two cases concern asymptomatic microscopic hematuria. We will be able to make a definite diagnosis via cystoscopy and tissue biopsy when the symptoms get worse.

In general, UC is a noninvasive method for detecting tumor cells in urine with high specificity. No noninvasive diagnostic method can fully take the place of histopathology. ${ }^{8}$ Among these noninvasive examinations, the FITC-CSNRDARRC ligand shows the closest results to histopathology.

In research on the CSNRDARRC ligand, ${ }^{19}$ fluorescenceactivated cell sorting and laser-scanning confocal microscopy were adopted. The results showed that the CSNRDARRC ligand had a dose-dependent affinity to bladder tumor cells, and that the binding site of the CSNRDARRC ligand was in the nuclei. As a screening test for bladder tumors, the CSNRDARRC ligand has characteristics of high affinity to bladder tumor cells. Therefore, the FITC-CSNRDARRC ligand has a certain rationality and feasibility for the study of bladder tumors.

In summary, the FITC-CSNRDARRC ligand shows superiority as a prognostic marker for patients suspected of having new or recurrent bladder tumors, has superior sensitivities and stable specificities, and is a noninvasive, low-cost, and simple experimental procedure. How this technology should be used to diagnose and monitor bladder tumors requires further study. In the future, we will investigate the role of the FITC-CSNRDARRC ligand in both the grading and staging of bladder tumors. It is believed that the

Table 3 Statistical analysis obtained by FITC-CSNRDARRC ligand, UC and FISH for the detection of bladder tumor

\begin{tabular}{|c|c|c|c|c|c|}
\hline & \multicolumn{4}{|c|}{$\underline{P \text {-value* (2-sided) }}$} & \multirow[t]{3}{*}{ Overall sensitivity } \\
\hline & \multicolumn{2}{|c|}{ Tumor grades } & \multicolumn{2}{|c|}{ Tumor stages } & \\
\hline & Low & High & pTa + pT I & pT2 - pT4 & \\
\hline UC vs FISH & 0.063 & 0.006 & 0.500 & 0.001 & 0.000 \\
\hline UC vs FITC-CSNRDARRC & 0.031 & 0.003 & 0.250 & 0.001 & 0.000 \\
\hline FISH vs FITC-CSNRDARRC & I & I & I & I & 0.625 \\
\hline
\end{tabular}

Note: *Statistically significant difference $(P<0.05)$, McNemar test.

Abbreviation: FISH, fluorescence in situ hybridization; UC urinary cytology. 
FITC-CSNRDARRC ligand will be a practical method for the detection and follow-up of bladder tumors and may aid in establishing the original diagnosis of the disease.

\section{Acknowledgments}

The research reported in this paper was carried out as part of a paper on the "Diagnosis of bladder tumor by targeting peptide mediated optical molecular imaging” (No 81172444), which was supported by the National Natural Science Foundation of China.

\section{Disclosure}

The authors report no conflicts of interest in this work.

\section{References}

1. Yoo JH, Suh B, Park TS, et al. Analysis of fluorescence in situ hybridization, mtDNA quantification, and mtDNA sequence for the detection of early bladder cancer. Cancer Genet and Cytogenet. 2010;198(2):107-117.

2. Tomera KM. NMP22 (R) BladderChek (R) Test: Point-of-care technology with life and money-saving potential. Expert Rev Mol Diagn. 2004;4(6):783-794.

3. Sylvester RJ. Natural history, recurrence, and progression in superficial bladder cancer. Scientific World Journal. 2006;6:2617-2625.

4. Luo JH, Hua WF, Rao HL, et al. Overexpression of EIF-5A2 predicts tumor recurrence and progression in $\mathrm{pTa} / \mathrm{pT} 1$ urothelial carcinoma of the bladder. Cancer Sci. 2009;100(5):896-902.

5. Sylvester RJ, van der Meijden APM, Oosterlinck W, et al. Predicting recurrence and progression in individual patients with stage Ta T1 bladder cancer using EORTC risk tables: a combined analysis of 2596 patients from seven EORTC trials. Eur Urol. 2006;49(3): 466-477.

6. Wang W, Shao RP, Wu QP, et al. Targeting gelatinases with a nearinfrared fluorescent cyclic His-Try-Gly-Phe peptide. Mol Imaging Biol. 2009;11(6):424-433.

7. Lee SM, Lee EJ, Hong HY, et al. Targeting bladder tumor cells in vivo and in the urine with a peptide identified by phage display. Mol Cancer Res. 2007;5(1):11-19.

8. Xu CL, Zeng QS, Hou JG, et al. Utility of a modality combining FISH and cytology in upper tract urothelial carcinoma detection in voided urine samples of Chinese patients. Urol. 2011;77(3):636-641.

9. Savic S, Bubendorf L. Fluorescence in situ hybridization: A new diagnostic dimension in cytology. Pathologe. 2007;28(5):384-392.

10. Sokolova IA, Halling KC, Jenkins RB, et al. The development of a multitarget, multicolor fluorescence in situ hybridization assay for the detection of urothelial carcinoma in urine. J Mol Diagn. 2000;2(3):116-123.
11. van Rhijn BWG, van der Poel HG, van der Kwast TH. Urine markers for bladder cancer surveillance: a systematic review. Eur Urol. 2005;47(6):736-748.

12. Akkad T, Brunner A, Pallwein L, et al. Fluorescence in situ hybridization for detecting upper urinary tract tumors: A preliminary report. Urol. 2007;70(4):753-757.

13. Mowatt G, Zhu S, Kilonzo M, et al. Systematic review of the clinical effectiveness and cost-effectiveness of photodynamic diagnosis and urine biomarkers (FISH, ImmunoCyt, NMP22) and cytology for the detection and follow-up of bladder cancer. Health Technol Assess. 2010;14(4):1-356.

14. Shariat SF, Lotan Y, Vickers A, et al. Statistical consideration for clinical biomarker research in bladder cancer. Urol Oncol. 2010;28(4):389-400.

15. Lotan Y, Shariat SF, Schmitz-Drager BJ, et al. Considerations on implementing diagnostic markers into clinical decision making in bladder cancer. Urol Oncol. 2010;28(4):441-448.

16. Laakkonen P, Zhang LL, Ruoslahti E. Peptide targeting of tumor lymph vessels. Ann N Y Acad Sci. 2008;1131:37-43.

17. Raitanen MP, Kaasinen E, Lukkarinen O, et al. Analysis of false-positive BTA stat test results in patients followed up for bladder cancer. Urol. 2001;57(4):680-684.

18. Inoue T, Nasu Y, Tsushima T, Miyaji Y, Murakami T, Kumon H. Chromosomal numerical aberrations of exfoliated cells in the urine detected by fluorescence in situ hybridization: Clinical implication for the detection of bladder cancer. Urol Res. 2000;28(1):57-61.

19. Zhao Yang, Xiao-feng Yang, Hao X-q. Peptides CSNRDARRC affinity and binding sites study of bladder cancer marker. J Contemp Urol. 2011; 3(2):105-107.

20. Marin-Aguilera M, Mengual L, Ribal MJ, et al. Utility of a multiprobe fluorescence in situ hybridization assay in the detection of superficial urothelial bladder cancer. Cancer Genet Cytogenet. 2007;173(2):131-135.

21. Song M-J, Lee H-M, Kim S-H. Clinical usefulness of fluorescence in situ hybridization for diagnosis and surveillance of bladder cancer. Cancer Genet Cytogenet. 15, 2010;198(2):144-150.

22. Guarnizo E, Pavlovich CP, Seiba M, Carlson DL, Vaughan ED, Sosa RE. Ureteroscopic biopsy of upper tract urothelial carcinoma: Improved diagnostic accuracy and histopathological considerations using a multibiopsy approach. J Urol. 2000;163(1):52-55.

23. Marin-Aguilera M, Menqual L, Ribal MJ, et al. Utility of fluorescence in situ hybridization as a non-invasive technique in the diagnosis of upper urinary tract urothelial carcinoma. Eur Urol. 2007;51(2):409-415.

24. Halling KC, Kipp BR. Bladder cancer detection using FISH (UroVysion assay). Adv Anat Pathol. 2008;15(5):279-286.
OncoTargets and Therapy

\section{Publish your work in this journal}

OncoTargets and Therapy is an international, peer-reviewed, open access journal focusing on the pathological basis of all cancers, potential targets for therapy and treatment protocols employed to improve the management of cancer patients. The journal also focuses on the impact of management programs and new therapeutic agents and protocols on

Submit your manuscript here: http://www.dovepress.com/oncotargets-and-therapy-journal

\section{Dovepress}

patient perspectives such as quality of life, adherence and satisfaction. The manuscript management system is completely online and includes a very quick and fair peer-review system, which is all easy to use. Visit http://www.dovepress.com/testimonials.php to read real quotes from published authors. 